Review

\title{
Opportunités d'une exploitation soutenue des plantes médicinales dans l'aménagement forestier
}

\author{
Nicole Marie GUEDJE ${ }^{1 *}$, Charles NTUNGWEN FOKUNANG ${ }^{1}$, Réné Bernadin \\ TAFOKOU JIOFACK ${ }^{2}$ et Robert FOGOU DONGMO ${ }^{3}$
}

\author{
${ }^{1}$ Département de Pharmacie et de Pharmacothérapeutique Africaine, Faculté de Médecine et des Sciences \\ Biomédicales, Université de Yaoundé I. B.P. : 1364 Yaoundé, Cameroun. \\ ${ }^{2}$ Musée Ecologique du Millénaire (Muséum d'histoire naturelle). B.P. : 8038, Yaoundé, Cameroun. \\ ${ }^{3}$ Centre Supérieur des Sciences de la Santé (CSSS), Université Catholique d'Afrique Centrale (UCAC). BP \\ 1110, Yaoundé, Cameroun. \\ Auteur correspondant,E-mail: manigue@hotmail.com /guenima@yahoo.fr; Tél: +237-99685850/+237- \\ 22664104 ; B.P. : 25645 Yaoundé, Cameroun.
}

\section{RESUME}

Les plantes médicinales constituent des ressources précieuses pour les populations rurales africaines. Plus de $80 \%$ de cette population s'en sert pour assurer leurs soins de santé. Ces plantes constituent également des ressources inestimables pour l'industrie pharmaceutique. Malgré ces énormes potentialités et perspectives, l'approvisionnement en plantes médicinales accuse un grave déclin dû à la déforestation et à la dégradation des écosystèmes. Le présent papier se propose d'examiner les opportunités d'une exploitation soutenue des plantes médicinales et autres produits forestiers non-ligneux afin de concilier les défis d'un accroissement des revenus et d'une conservation de la biodiversité. Une telle gestion pourrait aisément être intégrée dans les schémas généraux d'aménagement forestier, si elle prend en compte les différentes phases de cet aménagement, à savoir le choix des essences; le mode d'accès, les droits d'usage et l'inventaire des ressources, l'analyse et le choix des paramètres d'exploitation, le suivi et le contrôle de l'exploitation. Cette intégration comporte plusieurs opportunités, notamment la récolte d'un plus grand nombre de ressources, la réduction des coûts d'inventaire d'exploitation et d'aménagement, la conservation de la biodiversité et de l'environnement et la possibilité d'une orientation vers l'éco-certification des ressources. Une telle approche de gestion pourrait être mise en application dans le cadre des concessions forestières, de l'agroforesterie et de la foresterie communautaire.

(C) 2010 International Formulae Group. All rights reserved.

Mots clés : plantes médicinales, produits forestiers non-ligneux, gestion durable, conservation de la biodiversité.

\section{INTRODUCTION}

Tout au cours de l'histoire, les forêts ont été estimées pour l'abondance des biens qu'elles procurent : produits de subsistance et produits d'échange, aliments, médicaments, épices, résines, gommes, latex, gibier, bois de feu et, naturellement, bois d'œuvre avec tous ses dérivés. Parmi ces différents biens, les Produits Forestiers Non-Ligneux (PFNL) sont exploités à travers le monde sur presque 
quatre milliards d'hectares de forêts, soit environ $30 \%$ des terres émergées (FAO, 2005). Les plantes médicinales, une des principales composantes de ces PFNL, constituent des ressources précieuses pour la grande majorité des populations rurales des pays en développement. En Afrique, plus de $80 \%$ de la population rurale s'en sert pour assurer leurs soins de santé (Fokunang et al., 2010; Jiofack et al., 2009, 2010). L'enclavement des zones rurales, l'inexistence ou l'état rudimentaire des infrastructures sanitaires, le coût élevé des préparations pharmaceutiques, ainsi que la modicité des revenus des populations, font de ces plantes médicinales un atout majeur, parfois l'unique recours pour ces populations. A ce titre, l'OUA (Organisation de l'Unité Africaine, aujourd'hui Union Africaine), reconnaissant que la médecine traditionnelle est « le système de soins de santé le plus facilement accessible pour la plupart des Africains ruraux », a institué la période 2001-2010 « Décennie de la médecine traditionnelle en Afrique », afin de valoriser ce système de soins de santé fondamental. Par ailleurs, l'Organisation Mondiale de la Santé (OMS) estime que, dans le monde, plus de 4 milliards de personnes utilisent la pharmacopée traditionnelle à base de plantes médicinales pour leurs soins de santé primaire. En Asie du Sud, les traditions médicales comme l'ayurveda, l'unani, le siddha, l'amchi, l'homéopathie et la médecine chinoise mettent à contribution environ 9000 espèces végétales (Shore, 2004).

Les plantes médicinales constituent également des ressources inestimables pour l'industrie pharmaceutique. Selon une estimation de l'OMS, elles représentent environ $70 \%$ des matériaux de base des produits pharmaceutiques modernes. Environ $25 \%$ des médicaments produits et commercialisés dans le monde proviennent des plantes, et au moins 35000 espèces végétales sont ainsi utilisées à des fins médicales (Kasparek et al-Janabi, 2008). Depuis 150 ans, les plantes médicinales ont fourni à la pharmacie des médicaments très efficaces. De nouveaux médicaments ont été mis au point en recherchant les principes actifs de plantes médicinales qui, pour la plupart, étaient des plantes toxiques (Lévêque et Mounoudou, 2001). Avec les avancées du génie génétique et des biotechnologies en général, les gènes et les molécules produites par la canopée, les racines ou d'autres parties des arbres, ainsi que d'autres organismes forestiers (dont bactéries et champignons) suscitent depuis les années 1970 un intérêt croissant dans le secteur de la chimie et de l'industrie pharmaceutique. De nombreuses autres espèces sont actuellement explorées dans une perspective de leur valorisation dans divers domaines, notamment cosmétique, pharmaceutique et pharmacologie, agroindustrie, industrie (Heinrich et al., 2004).

Malgré ces énormes potentialités et perspectives, les ressources en plantes médicinales accusent un grave déclin en raison de la perte annuelle d'environ 6 millions d'hectares de terres ou de couvert végétal, due à la déforestation et à la dégradation des écosystèmes. La recherche de nouvelles molécules par les industries pharmaceutiques, une fois l'identification faite, se heurte à une difficulté majeure, celle de disposer suffisamment de matériel vivant pour en assurer l'exploitation. L'agriculture pérenne ou itinérante, l'extension des fermes et exploitations industrielles, la surexploitation ou la mauvaise gestion des ressources naturelles et de leur transformation résultant du commerce national, régional ou international, seraient quelques unes des principales causes de cette déforestation et importante dégradation des écosystèmes et des ressources (Wickens, 1991). A ces principales causes, s'ajoutent également d'autres, non moins importantes, notamment la faiblesse des investissements dans la mise en œuvre des plans d'aménagement, l'inadéquation des législations et des politiques nationales en matière d'exploitation, de gestion et de conservation des ressources, ainsi que l'insuffisance des connaissances sur ces ressources biologiques et la mise en œuvre de leur conservation. De multiples exemples montrent à cet égard que des milliers 
d'espèces de plantes médicinales (Prunus africana, Garcinia kola, Garcinia lucida, Siphonochilus aethiopicus, Warburgia salutaris) soumises à une intense exploitation, sont actuellement menacées de disparition à travers le monde (Diederichs et al., 2002; Cunningham et Mbenkum, 1993 ; Oldfield et al., 1998 ; Soehartono et Newton, 2001).

C'est ainsi qu'au regard de ces menaces, et suivant les directives de la Convention sur la Diversité Biologique (CDB), une exploitation commerciale des PFNL en général, et des plantes médicinales en particulier, sur une base socialement équitable et acceptable, économiquement viable, écologiquement durable et compatible avec l'aménagement forestier devient un impératif.

L'aménagement forestier, qui a jusqu'ici marginalisé le secteur de ces PFNL au profit de l'exploitation forestière, ne peut plus se focaliser ou se limiter à la seule production de bois d'œuvre. Il devrait de plus en plus tenir compte de l'impact des PFNL sur l'économie rurale, nationale et régionale, et leur rôle potentiel en matière d'amélioration des revenus et de conservation de la biodiversité.

En ce qui concerne les plantes médicinales et leur importance dans les systèmes de soins de santé et l'industrie pharmaceutique, l'aménagement devrait viser à promouvoir leur valorisation tout en maintenant, ou au mieux, en accroissant leur productivité. Le présent papier se propose d'examiner les opportunités d'une intégration de l'exploitation soutenue des PFNL en général, et des plantes médicinales en particulier, dans les schémas d'aménagement forestier, afin de concilier les défis d'un accroissement des revenus et d'une conservation de la biodiversité des massifs forestiers. Les principaux domaines d'application des stratégies de gestion de ces ressources sont également analysés. L'idée de gestion durable est au centre de la foresterie depuis son origine. Cependant, la signification de la notion de gestion durable appliquée à la foresterie a évolué de manière significative avec le temps (Eba'a Atyi, 2001). Dans le cadre de la présente étude, lorsque le concept de gestion durable s'applique à une espèce ou à sa population d'arbres, il intègre l'ensemble des mesures appliquées pour optimiser la production des organes récoltés et la pérennisation de l'espèce. La notion d'exploitation soutenue est entendue ici comme une utilisation actuelle des ressources, mais réalisée avec le souci de laisser aux générations futures, au moins autant de bénéfices qu'aux générations actuelles. On distingue généralement deux approches complémentaires pour la gestion durable des PFNL : (i) l'exploitation des ressources dans leur milieu naturel (in situ) et (ii) la culture des ressources dans les espaces agroforestiers (ex situ).

\section{Principales caractéristiques socio- économiques, biologiques et écologiques des plantes médicinales}

L'une des principales caractéristiques des forêts tropicales est sa biodiversité en matière de ressources ligneuses et nonligneuses. Cette biodiversité, qui fait la richesse et l'attrait de ces écosystèmes, est également le principal facteur responsable des faibles densités de tiges par hectare pour les essences considérées individuellement. Dans cet écosystème, il existe également une grande variabilité dans la dynamique de la phénologie, de la reproduction, de la régénération et de la croissance des espèces. Ces caractéristiques biologiques et écologiques, qui interagissent avec d'autres facteurs socio-économiques, humains et techniques, constituent des paramètres de poids qui déterminent l'aptitude des ressources à se prêter à une gestion durable.

\section{Caractéristiques biologiques et écologiques}

Sur le plan écologique, bien que chaque espèce présente des caractères biologiques qui lui soient propres (phénologie, biologie de reproduction, tempérament, structure diamétrique), plusieurs espèces adoptent des stratégies démographiques ou biologiques similaires. C'est d'ailleurs pourquoi certains auteurs (Whitmore, 1989; Peters, 1997) distinguent, partant de l'autoécologie des 
espèces, deux ou trois grands groupes écologiques: (i) le groupe des espèces climaciques (graines peu nombreuses, de grande taille, sans dormance; arbre à bois dense, à croissance lente et grande longévité), (ii) celui des espèces pionnières (graines de petite taille, dormantes et persistantes dans le sol ; arbres à bois moins dense, à croissance rapide et longévité très courte) et, accessoirement, (iii) celui des espèces de forêt secondaire. Les caractères biologiques des individus appartenant à une espèce et les caractéristiques démographiques des populations qu'ils constituent traduisent une adaptation à l'environnement et sont le résultat d'une évolution en interdépendance sous l'effet de la sélection naturelle. Ce qui a permis d'élaborer le concept de sélection $r$ et sélection $k$ (Pianka, 1970).

Les individus sélectionnés $r \quad$ ou stratèges $r$, sont des opportunistes à haut potentiel de colonisation qui constituent des populations tirant profit d'habitats et dont le comportement est instable, imprévisible ou éphémère. Ces individus sont généralement de petite taille et à maturité reproductive précoce. Ils produisent une descendance nombreuse, leurs graines présentent une dormance qui leur assure une viabilité à long terme et joue un rôle important dans la stratégie opportuniste qu'adoptent ces individus.

Les stratèges $k$ appartiennent par contre à des populations installées dans des habitats stables ou qui présentent des variations à caractère saisonnier, d'effectif constant, proches de la capacité de charge maximale de l'environnement et en équilibre avec ses ressources limitées (Degreef, 1998). Les individus sélectionnés $\mathrm{k}$ sont de taille relativement grande, ils produisent des graines en petit nombre et consentent une faible allocation de reproduction afin de favoriser leur survie, de sorte qu'ils aient une grande longévité.

En matière de stratégies de régénération naturelle, de nombreux mammifères sont impliqués dans la prédation/dispersion des graines ou des fruits en forêt, mais leur contribution quantitative pour la régénération est très inégale et parfois ambiguë (Debroux, 1998). Les différents avantages que procure la dispersion aux graines sont d'échapper à la destruction dépendante de la distance du semencier ou de la densité des conspécifiques, et d'atteindre des sites favorables, localisés aléatoirement ou de manière déterminée (Venable et Brown, 1993, in Debroux, 1998). Dans le même ordre d'idée, les résultats de Harms et Dalling (1997) montrent que les plantules d'espèces à larges graines réagissent relativement mieux à la prédation que les espèces à graines de petite taille.

A la lumière de l'importance de ces caractéristiques, nous nous accordons avec Nepstad et al. (1992), Peters (1997), pour considérer que les espèces de plantes médicinales et autres PFNL exploitées et disposant de caractéristiques biologiques et écologiques leur permettant de se reproduire et d'installer continuellement de nouveaux individus, sont celles qui présentent de meilleures perspectives pour une gestion durable. Ce sont notamment des espèces dont les fruits (Voacanga africana, Ricinus communis) ou les feuilles (Senna alata) font l'objet de récolte préférentiellement par rapport à la récolte des plantes entières ou de l'écorce par annelation complète des arbres, comme c'est le cas avec Alstonia boonei, Annickia clorantha, Prunus africana. Ce sont également des espèces à stratégies de régénération naturelle et stratégies démographiques efficaces (mécanismes de pollinisation et de dispersion efficaces, fort pouvoir germinatif, taux élevé de survie des semis, forte densité de peuplement, structure diamétrique présentant une allure décroissante des plus petits individus aux plus grands), comme c'est le cas avec Garcinia lucida. Ce sont enfin des espèces présentant une forte capacité de développement des mécanismes de survie tels que la réitération traumatique, la reconstitution des organes prélevés (feuilles, écorces, racines), comme c'est le cas avec Cecropia peltata, Garcinia lucida, Cola acuminata, Cola nitida. 


\section{Caractéristiques \\ socioculturelles \\ et techniques}

Sur le plan socioculturel et technique, de nombreux travaux ont démontré que l'importance sociale, culturelle et médicinale de multiples PFNL était variable ou considérable suivant leurs niches écologiques et suivant le contexte local ou l'environnement de leur exploitation (Wickens, 1991). Des études ont été conduites sur des aspects relatifs aux modes d'accès et la tenure foncière de ces PFNL (Tsague, 1995; Nguinguiri, 1999 ; Tchatat et al., 1999). Pour de multiples plantes médicinales, le libre accès à la ressource est le principal mode régissant généralement l'exploitation de ces ressources en forêt naturelle. Ce mode de libre accès a pour principal avantage de rendre la ressource accessible à tous les membres de la communauté. Cependant, l'inconvénient majeur est l'exploitation intensive ou incontrôlée des ressources, et dans ce cas, il devient difficile de maintenir ou d'assurer la pérennité et le renouvellement des ressources avec ce mode de régulation.

En matière d'utilisation traditionnelle des PFNL, des études réalisées par de Beer et McDermott (1989), Falconer (1990), la FAO (1991), proposent des monographies consacrées à la définition, à la classification, aux utilisations, à l'importance des PFNL. De multiples autres travaux abordent ces PFNL en tant qu'objets d'étude socio-économique pour montrer ou évaluer leur importance comme source de subsistance ou de revenus pour les populations rurales (van Andel, 2006; Chupezi et al., 2009, 2010; Cunningham et al., 2002; Dold et Cocks, 2002 ; Ndoye et al., 1998, 2001); Certains travaux abordent ces PFNL comme source de devises comparables à celles générées par l'exploitation du bois d'œuvre (Peters et al. 1989); ou comme une des alternatives pour un aménagement et une conservation des forêts et de la biodiversité (Kasparek et al-Janabi, 2008; Nepstad et Schwartzman, 1992 ; Panayotou et Ashton, 1992). D'autres études traitent de leur importance nutritionnelle, médicinale ou cosmétique (Adjanohoun et al.,
1996 ; Betti, 2001; Betti et Lejoly, 2009 ; FAO/COMIFAC, 2006; Vivien et Faure, 1996 ; Tabuti et al., 2003). Certains travaux se penchent sur des aspects relatifs à la structure de population ou à la productivité des arbres, à la dynamique de population, à la gestion et à la conservation des ressources (Chikamai et al., 2009 ; Emanuel et al., 2005 ; Ndangalasi et al., 2007).

En ce qui concerne l'exploitation de ces PFNL, les modalités de récolte (intensités, fréquences et techniques des prélèvements), qui varient suivant le type d'organe exploité, présentent cependant dans différentes régions tropicales, beaucoup de similarités pour les mêmes types d'organe récoltés, qu'ils appartiennent ou non à différentes espèces. Ces modalités d'exploitation constituent des caractéristiques techniques influençant la disponibilité des ressources et la survie des espèces. Ces caractéristiques, sont de ce fait, des éléments importants dans la détermination des paramètres d'une exploitation à faible impact des ressources en plantes médicinales et autres PFNL. Une stratégie adaptée à ces paramètres consistera à gérer les populations d'espèces exploitées, à travers principalement une meilleure régulation de l'accès aux ressources et une application des méthodes de prélèvement efficaces et à faible impact en milieu naturel (in situ).

L'exploitation destructrice ou à impact considérable regroupe les méthodes pour lesquelles d'importants volumes d'organes sont extraits des individus ou de la population d'arbres, des techniques de prélèvement entraînent l'élimination des individus exploités, l'intensité et la fréquence des prélèvements appliquées ne permettent pas un renouvellement de la ressource. L'annélation, le déracinement et l'abattage des arbres pour la récolte de l'écorce, des fruits, des feuilles ou des racines de diverses espèces ligneuses, sont quelques exemples illustrant ce type d'exploitation, appliqué généralement par des utilisateurs qui exercent de manière régulière ou périodique, la commercialisation de ces ressources. L'impact de ce type d'exploitation est une forte pression exercée sur la ressource. 
Des travaux indiquent que la pression de l'exploitation exercée par les populations humaines est la principale cause de la raréfaction ou de la disparition de nombreuses espèces végétales et animales (Siebert et Belsky, 1985 in Peters, 1996 ; Peters, 1997).

L'exploitation à faible impact regroupe les méthodes efficaces pour lesquelles les techniques de prélèvement n'entraînent pas l'élimination à court ou à long terme des individus exploités, l'intensité et les fréquences de prélèvements appliquées permettent le renouvellement de la ressource. Le ramassage des fruits et des graines de diverses espèces ligneuses forestières sous la couronne des arbres, le prélèvement partiel de l'écorce sur les arbres et dans une moindre mesure, l'extraction des sèves sur des arbres sur pied, sont quelques exemples qui illustrent ce type d'exploitation. Il est généralement appliqué par des utilisateurs qui exploitent ces ressources pour la satisfaction des besoins de subsistance et de santé de leur ménage. L'impact de ce type d'exploitation est négligeable sur l'abondance et l'existence de la ressource, en supposant pour le cas des graines ou des fruits, qu'une certaine proportion échappe à la récolte et à la prédation pour assurer la régénération des espèces.

A la lumière de cette analyse, il s'avère utile de fixer les principaux paramètres permettant de réaliser une extraction à faible impact des plantes médicinales. Pour cela, une bonne connaissance des caractéristiques socio-économiques, biologiques et écologiques des espèces est primordiale pour la détermination de ces paramètres d'exploitation soutenue. L'approche que nous préconisons, pour la détermination de ces paramètres ou stratégies, consiste à observer la dynamique de population des espèces exploitées, à tester différents niveaux de récolte et à impliquer les utilisateurs locaux de la ressource à ces processus d'observation et d'évaluation. Nous nous accordons avec Guedje et al. (2006) et Wickens (1991), pour reconnaître que des études spécifiques, localement circonscrites et basées sur une telle approche, permettent une meilleure connaissance des ressources et une évaluation de leur potentiel de gestion. Cette approche permet de substituer aux observations et mesures empiriques, des stratégies de conservation basées sur des données démographiques et des analyses objectives. De telles études trouvent de nombreuses applications dans la conservation d'espèces rares ou menacées d'extinction; elles sont indispensables pour l'élaboration des stratégies effectives de gestion durable des plantes médicinales et autres PFNL, ainsi que leur intégration dans des programmes d'aménagement forestier. Ceci est d'autant plus justifié que la nature, l'utilisation et l'importance de ces ressources sont étroitement liées aux conditions économiques, socioculturelles et écologiques du contexte local de ces ressources. De telles études de cas (Guedje, 2002; Guedje et al., 2003, 2007 ; Péres et al., 2003 ; Soehartono et Newton, 2001), se focalisant essentiellement sur les ressources exploitées massivement, permettent de mieux appréhender l'impact de leur exploitation sur la dynamique de population et de formuler des recommandations relatives à leur gestion de manière durable.

Il serait donc intéressant d'envisager de cette manière, l'étude spécifique des plantes médicinales dont la demande actuelle sur les marchés nationaux ou internationaux menace leur disponibilité à long terme. Il s'agit, pour ne citer que quelques exemples, de Prunus africana, Pausynistalia johimbe, Gnetum africanum, G. buchholzianum, Eremospatha macrocarpa et Garcinia kola au Cameroun (Sunderland et al., 1999; Wilkie, 1999); de Warburgia salutaris, Ocotea bullata et de Curtisia dentata en Afrique du Sud (Cunningham, 1990); de Mauritia flexuosa, Genipa americana, Euterpe precatoria et Chamaedorea spp. en Asie et en Amérique (Cabrera-Madrid et al., 1990, in Peters, 1996 ; Peters et al., 1989 ; Varquez et Gentry, 1989). Oeuvrant dans ce sens, la CITES (Convention on International Trade in Endangered Species of wild Fauna and Flora), la CBD 
(Convention on Biological Diversity) et TRAFFIC (Wildlife trade monitoring programme of WWF-World Wide Fund for Nature and IUCN-The World Conservation Union) mènent des actions dans le but d'identifier les espèces soumises à une plus forte pression dans le monde et de publier des recueils ou listes exhaustives, ainsi que des mesures ou directives pour la conservation de ces espèces menacées (Secretariat of the Convention on Biological Diversity, 2009).

Exploitation des plantes médicinales et accroissement des revenus

Importance économique

La diversification et l'accroissement des revenus au profit des humains constituent l'un des aspects les plus intéressants de l'aménagement forestier. De nombreux auteurs considèrent que l'exploitation sur une base soutenue des PFNL, dont les plantes médicinales, est une activité rentable comparable à l'exploitation forestière et pouvant contribuer à un accroissement des revenus au profit des populations locales (Awono et al., 2009 ; Ros Tonen et al., 1994). De nombreux travaux se sont attelés à évaluer la valeur des bénéfices pouvant être tirés des PFNL par hectare de forêt naturelle. Peters et al. (1989) ont estimé à $422 \$ /$ ha / an, le revenu net d'une exploitation soutenue des espèces forestières en Amazonie. Godoy et Lubowski (1992) ont estimé que la valeur nette des PFNL présents en forêt tropicale varie entre 0,75 et $420 \$ /$ ha / an. Lescuyer (comm. pers.) a estimé que la valeur nette des bénéfices tirés des prélèvements traditionnels des PFNL végétaux et animaux était de l'ordre de $1300 \mathrm{~F} \mathrm{CFA} \mathrm{/} \mathrm{ha} \mathrm{/} \mathrm{an} \mathrm{dans} \mathrm{l'Est-Cameroun.}$ Dans le sud de la Côte d'Ivoire, dans la forêt nationale de Yapo, composée de plantations et de peuplements naturels, les produits non ligneux ont rapporté, en 1987, environ 26000 dollars $\mathrm{EU}$, qui ont contribué à financer le coût de l'aménagement (Falconer, 1990). En ce qui concerne les espèces prises individuellement, Guedje et al. (comm. pers.) ont estimé que la valeur potentielle de l'écorce de Garcinia lucida était comprise entre 2633
F CFA / ha et $110734 \mathrm{~F} \mathrm{CFA} \mathrm{/} \mathrm{ha} \mathrm{au} \mathrm{niveau}$ des villages; et entre $12312 \mathrm{~F} \mathrm{CFA} \mathrm{/} \mathrm{ha} \mathrm{et}$ $517716 \mathrm{~F}$ CFA / ha au niveau des marchés urbains. Ingram et al. (2010) ont estimé à 709000 F CFA, le revenu moyen annuel obtenu de la commercialisation des bambous (Yushina alpina, Oxytenanthera abyssinica, Bambusa vulgaris) au Cameroun, avec des valeurs comprises entre 300000 et 12 millions de F CFA.

Les marchés et les débouchés existent, plus particulièrement pour les essences à propriété médicinale comme les graines de Strophanthus gratus, l'écorce de Pausinystalia johimbe. L'exportation hors du Cameroun, de Prunus africana, est estimée à 3000 tonnes de $\mathrm{m}^{3}$ d'écorce par an. Cette exportation génère environ 220 millions US\$ par an (Schröder, 2001). En Indonésie, des compagnies comme PT Jamu Air Mancur réalisent des revenus annuels de l'ordre de 10 millions US\$ et emploient 700 personnes, grâce à la demande sans cesse croissante des plantes médicinales dans les cités urbaines (FAO, 1995a). Plusieurs travaux mettent en évidence la contribution de ces ressources aux moyens d'existence des communautés rurales et urbaines. Sur les marchés de la Zone de Forêt Humide du Cameroun, le profit hebdomadaire moyen par commerçant pour l'ensemble des ventes de neuf principaux PFNL était de 8200 Francs CFA (16,50 dollars EU). Ce chiffre est supérieur au salaire minimal établi par le gouvernement pour les travailleurs urbains, qui était de 6500 Francs CFA (13 dollars EU) par semaine à la période de l'enquête (Péres et al., 2003). En Afrique du Sud, le commerce en plantes médicinales par les circuits informels, génèrent annuellement environ 35 millions de dollars EU au niveau des producteurs, et environ 280 millions de dollars EU au niveau des utilisateurs tels que les guérisseurs ou revendeurs de ces plantes médicinales (Chupezi et al., 2009).

En ce qui concerne plus spécifiquement l'industrie mondiale des plantes médicinales, celle-ci était évaluée en 2004, à plus de 60 milliards de dollars annuellement, représentant plus d'une augmentation de 
quatre cents pour cent depuis 1996. Ce marché continu de s'accroître avec presque $20 \%$ chaque année. En 1996, le réseau de suivi des échanges commerciaux TRAFFIC a estimé la valeur du marché mondial des plantes médicinales à US\$ 1,3 milliards. En 1998, le marché de détail total pour les plantes médicinales aux États-Unis a été estimé à 3,97 milliards de dollars, plus du double de l'estimation pour l'Amérique du Nord en 1996 (TRAFFIC, 1999). L'Egypte est le principal pays exportateur de plantes médicinales d'Afrique, et le cinquième exportateur de plantes médicinales au niveau mondial. $\mathrm{Au}$ début des années 1990, l'Egypte exportait 11250 tonnes de plantes médicinales par année, ayant une valeur de plus de US\$ 12 millions. En Afrique du Sud, la valeur des échanges commerciaux nationaux correspondant aux plantes médicinales est estimée à US\$ 6 ou 9 millions par année. L’on trouve des herbes médicinales provenant de l'Afrique du Sud en vente sur l'Internet. L'on considère que le marché interne pour les plantes médicinales est plus important que le marché d'exportation, puisque la grande majorité des africains consultent des guérisseurs traditionnels, autrement dit, des tradipraticiens (Andel, 2006).

Parmi les diverses et multiples plantes médicinales du Cameroun, quelques unes à effets cliniques établis, ont fait l'objet de protocole de recherche en laboratoire et sont également exportées. Il s'agit notamment de :

- Prunus africana, surexploitée dans le Mt Cameroun et ses environs, et dont les extraits d'écorce sont actifs dans le traitement de la prostatite, les capsules de ces extraits d'écorce sont vendues en Europe depuis plus de 40 ans; le coût de ce commerce se chiffre à plus de 150 millions de dollars par an (Cunningham et Mbenkum, 1993) ;

- $\quad$ Strophantus gratus, dont les graines contiennent de la strophantine qui est un alcaloïde cardiotonique; selon le Département forestier, le Cameroun fait un chiffre d'affaires de 175 millions de Francs CFA par an avec la commercialisation de cette espèce ;

- Pausinystalia johimbe, dont l'écorce contient la johimbine utilisée comme aphrodisiaque et vendue en comprimé sous la marque «YOCON » Johimbine HCL ;

- Ancistrocladus korupensis, dont les extraits ont de remarquables effets contre les virus HIV - 1 et HIV - 2 ; les feuilles et les branches ont également des propriétés antipaludiques.

Ces différents chiffres et estimations montrent l'importance et le potentiel économique de ces plantes médicinales, ainsi que leur contribution aux économies rurales et nationales. L'Allemagne importe chaque année, pour plus de 75 millions d'euros, des plantes médicinales, provenant majoritairement de pays en développement. La vente durable des plantes médicinales peut donc considérablement améliorer la balance commerciale de ces pays en voie de développement et représente un facteur important de développement. En effet, ces pays sont détenteurs d'une diversité biologique unique au monde, puisqu'ils abritent $90 \%$ du patrimoine génétique de la planète.

Sur le plan de la petite industrie, il existe de plus en plus des unités industrielles dans le secteur des plantes médicinales et autres PFNL à l'exemple de trois principaux laboratoires au Cameroun: (i) le laboratoire LOBOTHERA produisant le sirop HEPASOR utilisé pour le traitement de l'hépatite virale B ; (ii) le laboratoire KAMSU-KOM produisant entre autres le sirop POLAGASTRAL A-T-200 utilisé pour le traitement des ulcères gastriques; (iii) et enfin le laboratoire AFRICAPHARM, produisant le sirop Gama utilisé pour le traitement des hémorroïdes. Toutefois, il est important de se rappeler que nombre de ces industries, étaient ou sont encore à l'origine, constituées d'entreprises artisanales, qui forment un élément des stratégies de subsistance des 
ménages ruraux. Pour certains, ces activités ont conservé ce caractère, mais il ne faut cependant pas oublier que ces secteurs d'activités ont connu une croissance telle qu'ils produisent des revenus substantiels pour les responsables de ces entreprises. Ces entreprises de plantes médicinales, même si elles ne seront jamais près d'égaler la contribution économique de l'industrie du bois d'œuvre, peuvent jouer un rôle important dans l'économie rurale, à l'exemple de celui joué par l'ex-société Plantecam au Cameroun (Nkuinkeu, 1998). En outre, la filière d'exploitation et de commercialisation des plantes médicinales et autres PFNL présente d'importants atouts: elle dispose d'une diversité et d'une multitude d'espèces et de produits, elle est peu exigeante en intrants, en capitaux et en main d'œuvre.

\section{Une matière première diversifiée et variée}

Les plantes médicinales et autres PFNL regroupent une grande diversité d'espèces végétales ou animales, et une multitude d'organes de plante qui sont exploités. Prance et al. (1987) indiquent que $48,6 \%$ à 78,7\% du nombre total d'espèces recensées sur un hectare de forêt dense pour quatre différents groupes ethniques installés au Brésil, en Bolivie et au Venezuela (Amazonie) sont des espèces utiles pour les populations locales. Falconer (1990) a réalisé une synthèse de plus de 200 références relatives aux usages alimentaires, nutritionnels, médicinaux, sociaux et économiques des PFNL.

La flore camerounaise est riche de plus de 8000 espèces et le potentiel camerounais en plantes médicinales est très élevé. Le répertoire de ces plantes médicinales indique entre 600 et 800 espèces de plantes reconnues et utilisées dans la médecine traditionnelle et moderne (Adjanohoun et al., 1996; Nkongmeneck, 2007). La littérature concernant ces plantes médicinales est assez étendue, comprenant des études ethnobotaniques (Guedje et Fankap, 2001 ; Mapi, 1988 ; Noumi, 1984 ; Vivien et Faure, 1996), des études phytochimiques, toxicologiques et pharmacologiques (Kamtchouing et al., 2006; Kuete et al.,
2010 ; Ngameni et al., 2007). Les travaux de Betti (2001) traitent des usages traditionnels et de vulnérabilité des plantes médicinales en zones des forêts denses humides du Cameroun. Ceux de van Dijk (1999) recensent les plus importantes plantes médicinales par catégorie de maladie traitée dans la région de Bipindi-Akom II. Les traitements par les plantes sont connus et pratiqués par l'immense majorité de la population camerounaise et en particulier la population rurale, en plus de ceux utilisés par les guérisseurs traditionnels établis. La dévaluation du Franc CFA en 1994 a augmenté les prix des produits pharmaceutiques et a conduit ces populations, surtout les plus démunies, à se rabattre davantage sur les plantes médicinales pour se soigner.

Les organes récoltés sont également divers. La plante est rarement utilisée entière. Le plus souvent, il s'agit d'une partie de la plante: rhizome, bulbe, racine, parties aériennes, tige, écorce, bourgeon, feuille, sommité fleurie, fleur, pétale, fruit, graine, tégument de graine, exsudation de la plante. Différentes parties d'une même plante peuvent avoir des utilisations différentes. Ces plantes médicinales et autres PFNL comprennent une grande diversité de produits utiles, classés selon la FAO (2005) en fourrage ; en matière première destinées à la préparation de médicaments et/ou produits aromatiques (huiles essentielles, etc.); en matière première destinée à la production de colorants ou teintures; en matière première pour la fabrication d'ustensiles et d'objets d'artisanat, et pour la construction; en plantes ornementales ; exsudats (résines, latex, huiles essentielles, parfum, arômes, etc.); et autres produits végétaux (mousses, écorces).

Une filière peu exigeante en intrants et en capitaux

La filière d'exploitation et de commercialisation des plantes médicinales et autres PFNL est une entreprise peu exigeante en capitaux et en intrants. Les entreprises de plantes médicinales exigent une main-d'œuvre peu spécialisée, mais elles peuvent stimuler 
l'entreprenariat et aider à l'acquisition de compétences en affaires. Les études qui quantifient la part des intrants du ménage, l'affectation de la main-d'œuvre, les revenus et les coûts attribuables aux activités relatives aux plantes médicinales et autres PFNL sont bien rares. Il est cependant de plus en plus reconnu que l'exploitation de ces ressources a pour privilège d'être le principal secteur forestier où les populations locales tirent profit de l'exploitation des ressources forestières. Elle utilise une main d'œuvre peu qualifiée et bon marché, elle nécessite peu d'investissements, de capitaux, d'infrastructure et de matériels ou d'outils technologiques, comparativement à l'exploitation forestière ou agricole. De plus, elle permet la survie de nombreux ménages ruraux et urbains à revenus très faibles ou moyens, qui exploitent et commercialisent ces ressources. Il est important de reconnaître que ce mode d'exploitation à faible technologie, est également peu rentable et ne pourrait pas efficacement aider les populations à améliorer leur condition de vie. Les réflexions actuelles sont orientées vers l'amélioration de ces techniques et technologies locales afin de permettre aux populations de tirer des meilleurs bénéfices de l'exploitation des PFNL (Chicamai et al., 2009 ; Chupezi et al., 2010 ; Ezekiel, 2010; Koné et al., 2009; Leakey et al., 2007 ; Tchoundjeu et al., 2006).

\section{Les facteurs limitant de la filière}

En plus des faibles densités qui caractérisent les espèces forestières tropicales et des contraintes écologiques propres à quelques espèces (absence ou difficulté de régénération naturelle), d'autres facteurs limitent la rentabilité et la compétitivité des plantes médicinales et autres PFNL sur les marchés. Ce sont notamment:

- La saisonnalité et la variabilité annuelle de la production fruitière de ces espèces. Ces facteurs font en sorte que les activités relatives aux fruits et graines de ces ressources soient généralement saisonnières ou cycliques, certains produits ne pouvant être récoltés qu'à certaines périodes de l'année (Dijkman,
2001 ; Hagen et Fight, 1999). De ce fait, l'offre et les prix varient énormément en fonction des saisons. Dans de telles circonstances, les quelques individus produisant les fruits en dehors de la saison normale de fructification de l'espèce ont une plus grande valeur marchande.

- L'absence de technologies appropriées de conditionnement, de conservation et /ou de transformation. Les méthodes de conditionnement et de transformation restent dans la plupart des cas, essentiellement artisanales et très rudimentaires. On observe une perte d'importantes quantités de la production de fruits, de fleurs, de racines ou d'écorce et une forte pénurie pendant la période inter-saisonnière.

- Le maintien de la qualité des produits récoltés. Elle est fort variable suivant les espèces exploitées ou les techniques de récolte et de conservation utilisées.

- L'enclavement des zones de production et le mauvais état des routes. Dans la plupart des pays africains, les zones forestières productrices de plantes médicinales et autres PFNL sont généralement enclavées, de sorte que le transport constitue un véritable problème d'approvisionnement des centres urbains. Pendant les saisons de pluies, le transport en commun est presque inexistant car les routes sont en très mauvais état. Or cette saison de pluies correspond généralement à la saison de production des arbres fruitiers forestiers tropicaux.

La gestion des populations d'arbres in situ et la mise en œuvre des mesures complémentaires telle la domestication des espèces les plus intéressantes ex situ, peuvent permettre de contourner quelques-uns de ces facteurs limitants. Le développement d'industries ou des technologies de transformation et de conservation faciliterait la promotion des Médicaments Traditionnels Améliorés (MTA) pour la santé des populations. Ce développement permettrait également l'intégration de ces produits très 
appréciés et couramment utilisés par les populations dans les circuits modernes de distribution. Une mise au point des techniques de contrôle et d'amélioration de la qualité susciterait probablement plus d'intérêts auprès des opérateurs économiques et des usagers dans cette filière des plantes médicinales et autres PFNL. Bien évidemment d'autres facteurs, dont l'analyse dépasse le cadre du présent travail, constituent des aspects dont la mise en œuvre permettra de garantir le succès d'une exploitation commerciale et soutenue des plantes médicinales et autres PFNL. Ce sont notamment, la législation, les débouchés, le capital physique, les biens privés, les infrastructures économiques et sociales, les capitaux financiers.

Exploitation des plantes médicinales et autres PFNL et gestion des massifs forestiers

Systèmes traditionnels de gestion et directives prévues par la loi forestière

Traditionnellement, les populations rurales ont institué un certain nombre de pratiques qui concourent à préserver certaines espèces de plantes utiles en milieu naturel et à favoriser leur régénération. Ces systèmes traditionnels de gestion utilisent généralement des techniques simples, telles que le sarclage autour de ces espèces utiles, la plantation d'enrichissement ou la transplantation des semis. L'abattage sélectif des arbres lors de la création des champs ou des plantations et la préservation de certaines espèces utiles (plantes médicinales, fruitières ou espèces à bois d'œuvre pour la construction et l'artisanat) est une pratique traditionnelle très répandue dans les systèmes agricoles itinérants sur brûlis en Afrique centrale (Bahuchet et de Maret, 1994; Carrière et McKey, 1999). Certaines espèces très utiles font l'objet de soins particuliers en forêt naturelle, dans les jachères et les champs afin de favoriser leur croissance et leur régénération (Schnell, 1957, in Bonnéhin, 2000). La transplantation des graines ou des semis de la forêt dans les plantations constitue également une autre pratique traditionnelle très fréquente en milieu forestier (Friedberg, 1999). Les soins apportés aux espèces utiles en milieu naturel et/ou la mise en culture de ces espèces utiles par les populations locales constituent, selon plusieurs auteurs, les deux premières étapes du processus de domestication (Bonnéhin, 2000; Wiersum, 1996).

La gestion des ressources par les populations forestières de PapouasieNouvelle-Guinée a entraîné la formation d'un paysage de forêts jardinées et domestiquées (Grouble, 1989, in McKey et al., 1996). Au Brésil (Ilha das Onças), de nombreuses espèces fournissant des plantes médicinales, des fruits comestibles, du latex et autres PFNL ont été entretenues et maintenues par les populations rurales (Reis, 1995, in FAO, 1995b). Les communautés Dayak du Kalimantan occidental pratiquent les plantations d'enrichissement pour maintenir des densités commercialement intéressantes de Durio spp., de rotin et d'illipé dans leurs forêts (Peters, 1997). De même, les peuplements d'Euterpe oleracea aménagés par les paysans d'Amazonie orientale sont enrichis par l'insertion de jeunes manguiers et cacaoyers. Dans les forêts sacrées, lieux sacrés pour les traditions, les rites d'initiation, les croyances ancestrales et les interdits contribuent à protéger une part importante de la biodiversité malgré les pressions anthropiques (Juhé-Beaulaton et al., 2005 ; Kokou et al., 2005).

Devant les pressions de plus en plus fortes des institutions internationales et des organisations environnementales, de nombreux pays se sont attelés à élaborer des directives en matière de gestion des écosystèmes forestiers. Les recommandations en matière de gestion des diverses ressources présentes dans les massifs forestiers sont reconnues grâce à des dispositions prévues par les nouvelles lois forestières, notamment la loi forestière camerounaise $\mathrm{N}^{\circ} 94 / 01$ de janvier 1994 (MINEF, 1998). Plus spécifiquement, les lois et textes réglementaires qui fixent le cadre de l'aménagement des forêts au Cameroun sont entre autres : 
- $\quad$ Loi $N^{\circ}$ 94/01 du 20 janvier 1994, portant régime des forêts, de la faune et de la pêche ;

- Décret No 95/531/PM du 23 août 1995, portant application du régime des forêts ;

- Décret $\mathrm{N}^{\circ}$ 95/678/PM du 18 décembre 1995 instituant un cadre indicatif d'utilisation des terres en zone forestières méridionales ;

- Décision N $N^{\circ}$ 108/D/MINEF du 09 février 1998 portant application des normes d'intervention en milieu forestier en République du Cameroun

- Décision $N^{\circ}$ 1354/D/MINEF/CAB du 26 novembre 1999 fixant les procédures de classement du domaine forestier permanent de la République du Cameroun ;

- $\quad$ Arrêté $N^{\circ}$ 0222/A/MINEF du 25 mai 2001 fixant les procédures d'élaboration, d'approbation, de suivi et de contrôle de la mise en œuvre, des plans d'aménagement des forêts de production du domaine forestier permanent.

- Décret sur les études d'impact environnementales.

En ce qui concerne les Produits Forestiers Non-Ligneux (PFNL), la cueillette de plantes médicinales est régie par l'obtention préalable d'un permis d'exploitation des espèces forestières, dont les conditions et qualifications requises sont définies dans Forêts, faune et régimes de pêches (Loi No 94/01 du 20 janvier 1994) et dans les directives d'utilisation de ce régime (Décret No 94/436 du 23 août 1994). La remise de ce permis s'accompagne d'un rapport décrivant avec précision les méthodes de récolte, correspondant à chaque structure végétative à exploiter. La prise en compte des PFNL en matière de gestion forestière est faite au niveau de la conservation de la biodiversité camerounaise, réglementée par :

- $\quad$ la loi 94/01 du 20 janvier 1994 en ses titres II (articles 11 à 19), IV (articles 78 à 81) ;
- la loi $\mathrm{N}^{\circ}$ 96/12 du 05 août 1996 portant loi cadre sur l'environnement ;

- les normes d'intervention en milieu forestier ;

- l'arrêté 0222/A/MINEF du 25 mai 2001 fixant les procédures d'élaboration, d'approbation, de suivi et de contrôle de la mise en œuvre, des plans d'aménagement des forêts de production du domaine forestier permanent dans son chapitre traitant de l'affectation des terres à l'intérieur des concessions forestières.

Le Gouvernement a par la suite développé un certain nombre d'instruments nécessaires pour la gestion durable de ses ressources (MINFOF, 2005, 2006). Il s'agit entre autres, du Plan d'Action Forestier National - PAFN; du Plan de Zonage du Cameroun forestier méridional; du Plan National de Gestion de l'Environnement PNGE ; du Programme de Conservation et de Gestion de la Biodiversité au Cameroun PCGBC; du Programme Sectoriel Forêts et Environnement - Cameroun, avec ses cinq grandes composantes réparties en trois catégories :

- Une composante générale dont le but est la maîtrise de la situation de l'environnement lié au secteur forestier au Cameroun - 'Gestion Environnementale des Activités Forestières' (I) ;

- Trois composantes spécifiques qui visent la gestion des forêts de production, la gestion de la faune et des aires protégées et la gestion communautaire des ressources 'Aménagement des forêts de production $\mathrm{du}$ domaine forestier permanent et valorisation des produits forestiers' (II), 'Conservation de la biodiversité et valorisation des ressources fauniques' (III), 'Gestion communautaire des ressources forestières et fauniques' (IV) ; 
- Une composante transversale pour le renforcement des capacités des acteurs du secteur - 'Renforcement des capacités institutionnelles, formation et recherche'.

Dans cette logique, les activités relatives aux PFNL sont de plus en plus considérées comme une des possibilités de mise en valeur des massifs forestiers. Pour Peluso et Padoch (1996) in Warner (2000), satisfaire les exigences des populations locales sur une base durable devrait être le premier objectif de la gestion forestière, et cela devrait se traduire par des mécanismes de contrôle de l'exploitation. Cette option, de même que la mise en œuvre des nouvelles orientations en matière de conservation des écosystèmes tropicaux, sont défendues par de nombreuses institutions ou organisations. On peut citer particulièrement l'Organisation Internationale des Bois Tropicaux (OIBT), l'Union Mondiale pour la Conservation de la Nature (UICN), le Fond Mondial pour la Nature (WWF), le Wildlife Conservation Society (WCS), le Centre International de recherche forestière (CIFOR), le Fond mondial pour l'alimentation (FAO), la Banque Mondiale, le Programme Régional de l'Afrique Centrale pour l'Environnement (CARPE), etc.

Opportunités d'une intégration des plantes médicinales et autres PFNL dans la gestion forestière

L'exploitation domestique ou commerciale des plantes médicinales sur une base soutenue telle qu'elle a été argumentée dans ce papier et par plusieurs autres auteurs, pourrait aisément être intégrée dans les schémas généraux d'aménagement de diverses végétations ou espaces forestiers (FAO, 1995b, 2007; MINFOF, 2006 ; Ndangalasia, 2007 ; Peters, 1996 ; Ros-Tonen, 1999 ; Sunderland et al., 1999a; Wollenberg et Ingles, 1998). Elle participe aux différentes phases suivantes de cet aménagement:

- Le choix des espèces, la détermination des modes d'accès à la ressource et des droits d'usage. Le choix des espèces à exploiter est déterminé par leurs importances médicinales, socio- économiques et leurs potentialités ou aptitudes écologiques à la gestion durable ;

- L'inventaire des ressources. Il permet d'évaluer la densité et la structure diamétrique des espèces et d'estimer le potentiel de ces espèces, de même que la capacité de production de la végétation ou des massifs forestiers. Une bonne connaissance des ressources permet une meilleure planification des opérations d'exploitation ;

- Le choix des paramètres d'exploitation. Ce choix, basé sur les caractéristiques biologiques et écologiques des essences, permet de déterminer les fréquences et les intensités optimales d'exploitation pour chaque ressource, les techniques optimales de prélèvement et les catégories ou stades biologiques à exploiter ;

- Le suivi et le contrôle de l'exploitation. Ils permettent de s'assurer de l'application effective des paramètres d'exploitation, d'ajuster les prélèvements afin de maintenir des niveaux d'exploitation durable ;

- Les mesures complémentaires. Elles consistent à accroître ou à améliorer la capacité productive des ressources exploitées ou des massifs forestiers. En marge des opérations sylvicoles favorisant la croissance des espèces à bois d'œuvre, des soins, tels que ceux traditionnellement pratiqués par les populations riveraines et évoqués au paragraphe précédent, peuvent également être apportés aux espèces de plantes médicinales et autres PFNL en milieu naturel.

L'intégration des plantes médicinales et autres PFNL dans les plans d'aménagement des végétations ou des forêts comporte plusieurs opportunités :

- la récolte d'un plus grand nombre de ressources, permettant ainsi une exploitation plus rationnelle et une valorisation du couvert végétal ou massif forestier. L'intégration de l'exploitation des espèces médicinales et autres produits 
non-ligneux avec celle $\mathrm{du}$ bois est génératrice de profits à l'échelle locale et rend économiquement viable un rythme d'exploitation plus lent, et donc moins dévastateur sur le plan écologique ;

- La réduction des coûts d'inventaire d'exploitation et d'aménagement. Plusieurs espèces fournissant des produits médicinaux et autres PFNL sont également des essences à bois d'œuvre. Baillonella toxisperma, Bertholetia excelsa, Alstonia boonei, Poga oleosa, en sont quelques exemples (van Dijk 1999, Laird, 1999);

- La conservation des massifs forestiers et de l'environnement. Les espèces fournissant les produits médicinaux et autres PFNL font partie intégrante de l'ensemble des éléments de l'écosystème forestier qui permettent à la forêt de remplir durablement toutes ses fonctions. Ces espèces bénéficient généralement de quelques mesures de gestion qui constituent des moyens primaires de conservation par les populations intéressées par leurs produits ;

- La possibilité d'une orientation vers l'écocertification des plantes médicinales et autres PFNL. Plusieurs organisations et entreprises se sont engagées dans le processus de labellisation et d'écocertification des produits forestiers pour ouvrir de nouveaux marchés. Selon Dijkman (2001), il existe des espoirs de voir la Bolivie et bien d'autres pays encore tel que l'Inde (Bhattacharya et al., 2008), produire la certification des plantes médicinales et autres PFNL. Dans cette perspective, au Maroc, les Berbères considèrent l'arganier (Argania spinosa) comme «l'arbre de vie », et l'huile fournie par ses amandes est utilisée pour soigner de nombreuses maladies. La GTZ accompagne depuis de nombreuses années la production d'huile d'argan. Outre des mesures visant à préserver l'exploitation traditionnelle et la production artisanale d'huile, la GTZ soutient aussi l'introduction de structures d'organisation adaptées et de normes d'hygiène et a aidé les coopératives à obtenir la bio-certification (Kasparek et al-Janabi, 2008).

La prise en compte effective des plantes médicinales et autres PFNL dans la gestion des massifs forestiers n'est cependant pas une option très aisée dans cet écosystème hétérogène et complexe qu'est la forêt tropicale. Plusieurs raisons peuvent être avancées:

- La multiplicité des espèces fournissant les produits médicinaux et autres PFNL rend difficile l'intégration de toutes ces espèces dans l'aménagement forestier;

- Les types, les formes et les tailles des espèces médicinales et autres PFNL récoltés sont fort diversifiés et assez variés, rendant ainsi une mécanisation ou une normalisation des techniques de prélèvement bien difficile;

- Par opposition à la ressource ligneuse où la rotation des coupes et la possibilité sont les principaux paramètres d'exploitation et de gestion des essences, la détermination des paramètres d'exploitation et de gestion des plantes médicinales et autres PFNL doit être basée sur une bonne connaissance des caractéristiques socio-économiques, biologiques et écologiques des espèces exploitées. A ce titre, l'absence de connaissance sur les caractéristiques biologiques et écologiques de nombreuses espèces ne facilite pas toujours l'application des différentes étapes prévues dans un schéma global d'aménagement forestier.

Par ailleurs, les effets à long terme d'une telle intégration des espèces médicinales et autres PFNL dans l'aménagement forestier doivent être calculés avec soin. Les processus écologiques ne sont jamais statiques, et toute intervention sur un écosystème impose des tensions. Ainsi, toute action entreprise pour assurer la production soutenue d'un produit forestier spécifique ne peut qu'affecter en retour d'autres biotes. Il faut donc adopter une conduite équilibrée prévoyant un maximum 
d'avantages pour un minimum de bouleversement des composantes de l'écosystème (Wickens, 1991).

A la lumière de cette analyse, il serait souhaitable que l'exploitation soutenue et l'intégration dans l'aménagement forestier ne concernent donc principalement que les espèces médicinales et autres espèces de PFNL les plus importants d'un point de vue social et économique, et pour lesquels l'exploitation est de nature à menacer la disponibilité de la ressource. La gestion des autres espèces jugées moins importantes à ce moment-là, viendrait se greffer ou se substituer à l'exploitation des espèces traditionnelles lorsque la demande ou les potentialités de celles-ci sont promues ou reconnues.

\section{Les principaux domaines d'application Les concessions forestières}

Les dispositions prévues dans les nouvelles lois en Afrique centrale recommandent pour l'aménagement forestier durable, la rédaction d'un plan d'aménagement avant toute mise en exploitation d'un massif forestier (Nguinguiri, 1999). Le "zonage" fait référence à la diversité du territoire que l'on découpe en zones, suivant des critères (écologiques, démographiques) pour y intervenir en tenant compte de la spécificité de chaque zone (Clouet, 2000). Ce plan d'affectation des terres ou plan de zonage, consiste en une délimitation de l'espace rural et du domaine forestier permanent qui servira de lieux d'application des plans d'aménagement forestier (Tchatat, 2001). Des schémas directeurs (étape du processus qui suit le plan de zonage) et des plans d'aménagement sont élaborés pour différents massifs forestiers du Cameroun (Côté, 1993 ; Fines et al., 2001a, 2001b ; Poulin Thériault, 1995 ; UICN, 1997).

$\mathrm{Au}$ Cameroun, les concessions forestières attribuées couvrent $42,95 \%$ des 14 millions d'ha qui font l'objet du plan d'affectation des terres forestières du Cameroun méridional. Bien que l'objectif principal de ces concessions forestières soit la production du bois d'oeuvre, ces massifs forestiers sont utilisés depuis longtemps par les populations locales pour satisfaire leurs besoins de subsistance et pour améliorer leurs revenus à travers l'exploitation des PFNL tels que les plantes médicinales, les plantes comestibles, les chenilles ou le gibier. La politique forestière recommande une gestion intégrée de l'ensemble des ressources (ligneuses et non ligneuses) qui se trouvent dans la forêt permanente et particulièrement dans les concessions forestières pour optimiser les avantages et les revenus de l'état (propriétaire des ressources forestières), des concessionnaires et des populations locales (FAO, 2007).

Dans le cadre de l'élaboration du plan d'aménagement du massif forestier de la région de Bipindi-Akom II, Fines et al. (2001a, 2001b) ont considéré ce massif comme une forêt pilote gérée par un organisme étatique en étroite collaboration avec la population locale. Il était prévu de signer un accord avec une compagnie forestière en vue de l'exploitation du bois d'œuvre. Le plan d'affectation des terres de ce massif forestier à usage multiple a été élaboré ainsi qu'il suit : série de production (bois d'œuvre et PFNL): 14421 ha (80\% du massif); série de protection: 2151 ha $(12 \%)$; série de conservation: 1106 ha $(6 \%)$ et série de recherche: 356 ha $(2 \%)$. Les activités de récolte des PFNL sont interdites dans les séries de conservation et de recherche. Elles sont tolérées dans les séries de protection et autorisées dans les séries de production, aussi longtemps que les techniques de prélèvement ne compromettront pas la survie des individus. Les stratégies de gestion des PFNL telles qu'envisagées dans cette étude pourront être mises en application dans les concessions forestières ou dans le cadre de ces séries de protection et de production. Leur application pourra également être plus efficace par le biais de l'agroforesterie et de la foresterie communautaire, qui sont les deux principaux cadres ou systèmes déjà fonctionnels et institutionnellement reconnus, permettant de 
faire participer directement les populations rurales à la gestion des ressources forestières.

\section{L'agroforesterie}

L'agroforesterie désigne les modes et techniques de mise en valeur des terres dans lesquels les végétaux ligneux pérennes sont délibérément associés dans une même parcelle à des cultures herbacées ou à l'élevage, soit spontanément selon un certain agencement dans l'espace, soit en succession dans le temps. Par opposition aux systèmes simples d'utilisation des terres comme les cultures vivrières et les plantations d'arbres, l'agroforesterie est un système complexe qui assure la production de plusieurs intrants et vise ainsi une grande couverture des besoins ou une diversification des produits et des revenus du planteur (Belsky, 1993, in Rice et Greenberg, 2000).

L'agroforesterie représente ainsi un système flexible par lequel les plantes médicinales et autres espèces fournissant les PFNL peuvent graduellement être domestiqués et gérés en se conformant aux pratiques locales en vigueur. Elle est préconisée comme une solution potentielle pour assurer la subsistance des populations forestières tropicales, face à la déforestation de cet écosystème (Leakey, 1998, in Tchoundjeu et al., 1999). L'objectif majeur est de développer des formes durables d'utilisation des terres qui pourront améliorer la productivité agricole en même temps que le bien-être des communautés. De plus, les plantes médicinales s'intègrent aisément aux cultures traditionnelles, comme le maïs, le haricot et les cultures maraîchères. Le décalage des récoltes dans le temps permet aux exploitants de mieux échelonner leurs recettes sur l'ensemble de l'année. Des exemples réussis de domestication des PFNL en général, et des plantes médicinales en particulier, sont courants, avec notamment, l'exemple du safou, Dacryodes edulis (Simons et Leakey, 2004); de la mangue sauvage, Irvingia gabonensis (Atangana et al., 2002); de l'okok, Gnetum africanum (Shiembo, 1999); de la marula, Sclerocarya birrea subsp caffra (Leakey, 2005); du jujubier, Ziziphus mauritiana (Koné et al., 2009); du joyimbe, Pausinystalia johimbe (Tchoundjeu et al., 2004); ou encore de Prunus africana (Avana et al., 2005; Tchoundjeu et al., 2002).

Les principaux points faibles de l'agroforesterie sont, comme ceux recensés dans le cadre de la domestication des espèces ligneuses, les restrictions foncières et le manque de terre cultivable. Nous nous accordons avec 1'UICN/Union Européenne (1998) pour préconiser que le développement de stratégies intégrées de production agroforestière et de valorisation des PFNL devra être recherché en priorité dans les périphéries urbaines et les zones rurales très peuplées; Dans ces zones où les populations ont besoin de produire des quantités de plus en plus grandes sur des espaces de moins en moins restreints, l'adoption des technologies agroforestières innovantes est assez facile.

\section{La foresterie communautaire}

- Dispositions prévues par la loi forestière

Les dispositions relatives à la création des forêts communautaires en Afrique centrale entendent répondre à la volonté de décentraliser la gestion des ressources forestières. Au Cameroun, l'illustration la plus visible du concept de gestion participative est le transfert d'une partie des attributs de "propriétaire de la forêt" de l'Etat vers les populations, en leur reconnaissant le droit de gérer de façon autonome une portion du domaine forestier non permanent (Guedje et al., 2008 ; Milol, 1999).

$$
\mathrm{La} \text { décision }
$$

$\mathrm{N}^{\circ}$ 0098/D/MINFOF/SG/DF/SDFC du 02 février 2009 portant adoption du document intitulé «Manuel de procédure d'acquisition et des normes de gestion des forêts communautaires » précise les étapes d'obtention d'une forêt communautaire au Cameroun qui consistent entre autres à l'information et sensibilisation des parties prenantes (1), au choix de l'entité juridique (2), à la réunion de concertation 3), à l'élaboration et à la soumission du dossier d'attribution d'une forêt communautaire (4), à l'élaboration du plan simple de gestion et de 
la convention définitive de gestion (5), à la mise en œuvre du plan simple de gestion et de la convention définitive de gestion (6), à la surveillance, au contrôle, au suivi de la forêt communautaire (7) et à la fiscalité (8).

Les articles 37 et 38 de la loi forestière du 20 janvier 1994 (MINEF, 1998), précisent les modalités d'attribution et de mise en application des conventions de gestion des forêts communautaires. La gestion de ces forêts relève des communautés villageoises concernées avec l'assistance technique de l'administration des forêts. Grâce à une convention de gestion, les communautés villageoises se voient transmises le droit de bénéficier, pour un temps limité par cette convention, de la gestion des fruits de l'exploitation de la forêt attribuée.

\section{- Expériences de création des forêts communautaires}

Des expériences de création de forêt communautaire sont engagées dans la perspective de traduire le processus légal en une démarche pratique d'intervention (Nguinguiri, 1999). En Amérique du Nord, des initiatives de foresterie communautaire visent à procurer, à travers la bonne intendance des forêts, de nouveaux moyens de subsistance aux communautés forestières pauvres qui dépendaient précédemment de l'exploitation forestière (Danks, 2000). Au Cameroun, ces expériences ont été menées à travers plusieurs opérations qui sont entre autres le projet API-Dimako, le Programme APFT/Gestion périphérique du Dja. Le projet API de Dimako, lancé en 1992 pour une durée de trois ans, a focalisé son attention sur la validité du zonage des forêts mis en place par l'administration camerounaise des Eaux et Forêts (Pénelon, 1996). Le Projet Forêts Communautaires / Gestion périphérie nord de la Réserve de faune du Dja a envisagé de tester par une approche scientifique, la mise en place des forêts communautaires telles que prévues par la loi forestière, de conclure les premières conventions de gestion entre les communautés pilotes et l'administration, et d'élaborer un plan d'aménagement de la zone entière en tant que zone tampon de la Réserve en collaboration avec l'exploitant forestier et les projets qui y interviennent (Nguenang et Feteke, 2000). La superficie maximale d'une forêt communautaire est fixée à 5000 ha par la loi forestière au Cameroun. Les plantes médicinales et autres PFNL se rencontrent dans diverses et différentes communautés végétales faisant bien partie intégrante des massifs forestiers à classer comme forêts communautaires dans les régions où les populations en font la demande.

Les initiatives de foresterie qui soutiennent l'accès aux ressources, la prise de décisions participatives et l'équité, aident à renforcer la sensation de bien-être, en particulier celle des pauvres (Warner, 2000). Delvingt et al. (2000) considèrent que les forêts communautaires constituent aujourd'hui un des rares moyens qui permettent de concilier le développement des populations locales et la pérennité des ressources forestières, en favorisant la gestion des forêts par et au profit des populations elles-mêmes. Un projet lancé en 1992 par le Gouvernement népalais avec l'appui du FIDA (Fonds International de Développement Agricole) et de la FAO, démontre le potentiel d'un système associant foresterie communautaire et foresterie à bail, comme stratégie de lutte contre la pauvreté et la remise en état de l'environnement au Népal (Ohler, GCP/NEP/052/NET, in Malla, 2000). Au niveau des revenus générés dans le cadre de la foresterie communautaire, Subedi (1999) in Malla (2000) indique que dans quelques zones du Népal, les ménages tirent des forêts communautaires, jusqu'à un quart de leur revenu total, de la vente des PFNL sur les marchés.

Comme pour la création d'aires
protégées intégrant l'homme dans
l'écosystème forestier, la création des forêts communautaires est cependant une expérience assez récente pour en tirer des conclusions générales quant à son succès. A ce titre, une analyse, faite par Guedje et al. (2008), à l'échelle des économies familiales, de la contribution effective de la gestion de ces 
forêts communautaires à l'amélioration des conditions de vie des populations du Sud Cameroun, fait ressortir de manière globale que les revenus financiers générés dans la phase actuelle de l'exploitation des forêts communautaires sont encore insuffisants pour amorcer un véritable développement des communautés villageoises bénéficiaires. Pour abonder dans le même sens, Milol (1999) avait déjà souligné que le principe posé par le législateur camerounais était confronté à de nombreuses difficultés d'application. Son étude de cas mettait en scène trois logiques. Celle d'un texte législatif qui aménage une décentralisation de la gestion du patrimoine forestier en faveur des communautés villageoises. Celle d'une administration visiblement réticente à promouvoir des forêts communautaires, mais qui est liée par les injonctions des institutions monétaires internationales prônant ce type de gestion participative comme technique de gestion durable. Et celle enfin d'une communauté villageoise en proie à de nombreux conflits internes liés aux modalités de gestion du projet de foresterie communautaire. A la lumière de ces analyses, il serait souhaitable de tirer les leçons des expériences en cours concernant la création et la légalisation des terroirs des populations forestières avant de pouvoir vulgariser et multiplier ces initiatives types sur l'ensemble des massifs forestiers. Quelques unes des recommandations préconisent la simplification du Plan Simple de Gestion de ces forêts communautaires afin de faciliter son appropriation et sa mise en œuvre par les communautés locales, qui n'ont pas toujours l'expertise technique requise. Egalement le renforcement des capacités de décision, de financement et de gestion des revenus de ces communautés locales, en s'inspirant de leurs modes de fonctionnement, de leurs associations endogènes et de leur savoir-faire, afin de faire d'elles les véritables maîtres d'ouvrage de ce processus de foresterie communautaire (Guedje et al., 2008).

\section{Conclusion}

La garantie d'une gestion durable des plantes médicinales et autres PFNL compatible avec l'aménagement forestier dépend non seulement des ressources qui s'y trouvent, mais également des acteurs qui les utilisent et des différentes formes d'utilisation de ces ressources. L'analyse des caractéristiques biologiques, écologiques, socio-économiques montre que ceux-ci constituent des paramètres de poids qui déterminent l'aptitude des ressources à se prêter à une gestion durable. Les espèces médicinales et autres PFNL exploitées et disposant de caractéristiques biologiques et écologiques leur permettant de recruter et d'installer continuellement de nouveaux individus sont celles qui présentent de meilleures perspectives pour une gestion durable.

La diversification et l'accroissement des revenus au profit des humains constituent l'un des aspects les plus intéressants de l'aménagement forestier. La commercialisation des plantes médicinales et autres Produits Forestiers Non-Ligneux génère des revenus qui représentent une contribution importante aux économies nationales. En plus, la filière d'exploitation et de commercialisation des plantes médicinales et autres PFNL présente d'importants atouts: elle dispose d'une diversité et d'une multitude de produits, elle est peu exigeante en intrants et en capitaux, elle utilise une main-d'œuvre peu qualifiée et bon marché, elle nécessite peu d'investissements, d'infrastructure et de matériels ou d'outils technologiques, comparativement à l'exploitation forestière.

$$
\text { L'exploitation domestique ou }
$$

commerciale de ces ressources sur une base soutenue telle qu'elle est argumentée dans la présente étude, peut aisément être intégrée dans les schémas généraux d'aménagement forestier, en participant à différentes phases de cet aménagement (choix des essences, mode d'accès et droits d'usage, inventaires des ressources, choix des paramètres d'exploitation, suivi et contrôle de l'exploitation). Cette intégration comporte 
plusieurs opportunités, notamment la récolte d'un plus grand nombre d'espèces, la réduction des coûts d'inventaire d'exploitation et d'aménagement, la conservation de la biodiversité et de l'environnement. Les stratégies de gestion des ressources telles qu'envisagées dans cette étude pourront être mises en application dans le cadre des concessions forestières. Leur application pourra également être plus efficace par le biais de l'agroforesterie et de la foresterie communautaire, qui sont les deux principaux cadres ou systèmes déjà fonctionnels et institutionnellement reconnus, permettant de faire participer directement les populations rurales à la gestion des ressources forestières.

Cependant, il serait souhaitable que l'exploitation soutenue des plantes médicinales et autres PFNL et leur intégration dans l'aménagement forestier ne concernent donc principalement que les espèces les plus intéressantes d'un point de vue médicinal, social et économique, et pour lesquels l'exploitation est de nature à menacer la disponibilité de la ressource. La gestion des autres espèces jugées moins importantes actuellement, viendrait se greffer ou se substituer à l'exploitation des espèces traditionnelles lorsque la demande ou les potentialités de celles-ci sont promues ou reconnues. De même, pour plus d'efficacité, le développement de stratégies intégrées de production agroforestière et de valorisation des plantes médicinales et autres PFNL devra être recherché en priorité dans les périphéries urbaines et les zones rurales très peuplées. En ce qui concerne les forêts communautaires, l'expérience est assez récente pour en tirer des conclusions générales quant à son succès. Bien évidemment d'autres facteurs, dont l'analyse dépasse le cadre du présent travail, constituent d'autres importants aspects dont la mise en ouvre permettra de garantir le succès d'une exploitation commerciale et soutenue des plantes médicinales et autres PFNL, compatible avec l'aménagement forestier et la génération des revenus. Ce sont notamment, les aspects concernant le développement des normes de durabilité pour la collecte des plantes médicinales; l'actualisation des connaissances des médecines traditionnelles à la lumière des connaissances scientifiques les plus récentes, suivant les recommandations de l'O.M.S. (Organisation Mondiale de la Santé); le développement des technologies d'extraction, de transformation, de conditionnement et de valorisation de ces plantes médicinales; la mise en oeuvre effective du plan stratégique de développement et d'intégration de la médecine traditionnelle; la mise en œuvre effective des mécanismes de partage équitable des avantages découlant de l'utilisation des connaissances, innovations et pratiques traditionnelles intéressant la conservation de la diversité biologique et l'utilisation durable des plantes médicinales tels que préconisés par la Convention sur la Diversité Biologique (CDB).

\section{BIBLIOGRAPHIE}

Adjanohoun EJ, Aboubakar N, Dramane K, Ebot ME, Ekpere IA, Enow-Orock EG, Focho D, Gibile ZO, Kamany A, Kamsu Kom J, Keita A, Mbenkum T, Mbi CN, Mbiele AL, Mbome IL, Mubiru NK, Nancy WL, Nkongmeneck B, Satabie, Sofowora A, Tamze V, Wirmum CK. 1996. Traditional Medecine and Pharmacopoeia : Contribution to Ethnobotanical and Floristic Studies in Cameroon. OUA/CSTR : Lagos, Nigeria.

Atangana AR, Ukafor V, Anegbeh PO, Asaah E, Tchoundjeu Z, Usoro C, Fondoun JM, Ndoumbe M, Leakey RRB. 2002. Domestication of Irvingia gabonensis: 2 . The Selection of multiple traits for potential cultivars from Cameroon and Nigeria. Agroforestry Systems, 55: 221229.

Avana TML, Tchoundjeu Z, Bell JM. 2005. Domestication de Prunus africana (Hook f.) Kalkam : Une stratégie pour la gestion durable des ressources d'une plante médicinale menacée d'extinction. Cameroon Journal of Ethnobotany, 1(1): $31-40$. 
Awono A, Manirakiza D, Ingram V. 2009. Mobilisation et Renforcement des Capacités des Petites et Moyennes Entreprises Impliquées dans les Filières des Produits Forestiers Non Ligneux en Afrique Centrale. CIFOR: Bogota. Available online at Cameroun. http://cameroun-foret.com/fr/ bibliotheque/10666

Bahuchet S, de Maret P. 1994. Situation des populations indigènes des forêts denses et humides. Rapport pour la DG VIII de la Commission Européenne : Luxembourg.

Bhattacharya P, Prasad R, Bhattacharyya R, Asokan A. 2008. Vers la certification des plantes médicinales et aromatiques sauvages dans quatre États de l'Inde. Unasylva, 230(59): 35-44.

Betti JL. 2001. Usages traditionnels et vulnérabilité des plantes médicinales en zones des forêts denses humides du Cameroun (Réserve de Biosphère du Dja et Yaoundé). Thèse de doctorat, Univ. Libre de Bruxelles, Bruxelles.

Betti JL, Lejoly J. 2009. Contribution to the knowledge of medicinal plants of the Dja Biosphere Reserve, Cameroon: Plants used for treating jaundice. Journal of Medicinal Plants Research, 3(12): 10561065. Available online at http://www.academicjournals.org/jmpr

Bonnéhin L. 2000. Domestication paysanne des arbres fruitiers forestiers. Cas de Coula edulis Baill., Olacaceae, et de Tieghmella heckelii Pierre ex A. Chev., Sapotaceae, autour du Parc National de Taï, Côte d'Ivoire. Thèse de doctorat, Université de Wageningen, Wageningen, p. 139.

Carrière S, McKey D. 1999. Les arbres orphelins des champs vivriers. Etude de l'abattage sélectif chez les Ntumu et de son impact sur la régénération de la forêt du Sud-Cameroun. In L'Homme et la Forêt Tropicale, Bahuchet S, Bley D, Pagezy H, Vernazza-Licht N (eds). Société d'Ecologie Humaine ; 255-266.

Chikamai B, Tchatat M, Tieguhong JC, Ndoye O. 2009. Forest Management for
Non-Wood Forest Products and Services in Sub-Saharan Africa. Discov. Innov., 21(1): 50 - 59 .

Chupezi TJ, Ndoye O, Tchatat M, Chikamai B. 2009. Processing and Marketing of Non-wood Forest Products: Potential Impacts and Challenges in Africa. Discov. Innov., 21(1): $60-65$.

Chupezi TJ, Ndoye O, Grouvels S, Useni Kembolo M, Asseng Ze A. 2010. Small scale forestry and non-wood forest products enterprise development for poverty alleviation in Central Africa. In Small Scale Forestry in a changing World: Opportunities and Challenges and the Role of Extention and Technology Transfert, Medved M (ed). Proceedings de la Conférence, 06 - 12 juin 2010, Bled, Slovenia. Slovenian Forestry Institute / Slovenia Forest Service : Bled; $87-101$.

Clouet Y. 2000. Le zonage à dire d'acteurs: méthode et perspectives. Bois For. Trop., 265(3): 45-59.

Côté S. 1993. Plan de zonage du Cameroun forestier méridional, objectifs, méthodologie, plan de zonage préliminaire. MINEF-ACDI-PTI : République du Cameroun.

Cunningham AB. 1990. Man and medicines: the exploitation and conservation of traditional Zulu medicinal plants. In Proceedings of the 1988 Aetfat Congress, Mitteilungen aus dem Institut fur allegemeine Botanik (eds.). Hamburg; 23: 979 - 990.

Cunningham AB, Mbenkum FT. 1993. Sustainability of harvesting Prunus africana Bark in Cameroon: a medicinal plant in International Trade. People and Plants Working Paper no 2, Division of Ecological Sciences. UNESCO: Paris France.

Cunningham AB, Ayuk E, Franzel S, Duguma B, Asanga C. 2002. An economic evaluation of medicinal tree cultivation: Prunus africana in Cameroon. People and Plants working paper 10. UNESCO : Paris. Available online at: 
http://www.rbgkew.org.uk/peopleplants/ wp/wp10/index.html

Danks C. 2000. Initiatives de foresterie communautaire pour la création des moyens de subsistance durables en milieu rural: un exemple en Amérique du Nord. Unasylva, 202(51): 53-63. Available online at http//www.fao.org/DOCREP/ X7273F/X7273f02.htm

de Beer JH, McDermott MJ. 1989. The Economic Value of Non-timber Forest Products in Southeast Asia. IUCN: Amsterdam the Netherlands.

Debroux L. 1998. L'aménagement des forêts tropicales fondé sur la gestion des populations d'arbres: l'exemple du moabi (Baillonella toxisperma Pierre) dans la forêt du Dja, Cameroun. Thèse de doctorat, Fac. Univer. Sciences Agronom. Gembloux, Gembloux, Belgium.

Degreef J. 1998. Développement d'un modèle démographique et applications à la conservation in situ de populations sauvages de haricot de Lima (Phaseolus lunatus L.) dans la vallée centrale du Costa Rica. Thèse de doctorat, Fac. Univer. Sciences Agronom. Gembloux. Gembloux, Belgium.

Delvingt W, Dethier M, Milol CA, Vermeulen C. 2000. La mise en place d'une forêt communautaire: une action raisonnée. In Les Peuples des Forêts Tropicales Aujourd'hui, Bahuchet S, de Maret $\mathrm{P}$ (eds.). APFT : Bruxelles ; 115-140.

Diederichs N, Geldenhuys C, Mitchell D. 2002. The first legal harvesters of protected medicinal plants in South Africa. Science in Africa - Africa's First On-Line Science Magazine. http://www.scienceinafrica.co.zo/2002/no vember/bark.htm

van Dijk JFW. 1999. Non-Timber Forest Products in the Bipindi-Akom II region, Cameroon: a socio-economic and ecological assessment. TropenbosCameroon Series 1, The Tropenbos Cameroon Programme: Kribi, Cameroon.

Dijkman W. 2001. NTFPs: an instrument in sustainable development? ETFRN News,
32: 5-7. Available online at http://en.wikipedia.org/wiki/Nontimber_forest_products

Dold AP, Cocks ML. 2002. The trade in medicinal plants in the Eastern Cape Province, South Africa. South African Journal of Science, 98: 589 - 597.

Emanuel PL, Shackleton CM, Baxter JS. 2005. Modelling the sustainable harvest of Sclerocarya birrea subsp. caffra fruits in the South African lowveld. Forest Ecology and Management, 214: 91-103.

Eba'a Atyi R. 2001. Principes et concepts essentiels en aménagement forestier. In Sustainable management of African rain forest, Foahom B, Jonkers WBI, Nkwi PN, Schimidt P, Tchatat M (eds). Seminar proceedings held in Kribi, Cameroon, November 1999. Part I. Workshop. The Tropenbos Foundation: Wageningen; 3-11.

Ezekiel A. 2010. Viable options and factors in consideration for low cost vegetative propagation of tropical trees. Int. J. Bot., 6: 187-193.

Falconer J. 1990. The Major Significance of Minor Forest Product; The local use and value of forest in the West African humid forest zone. Community Forestry Note, No. 6. F.A.O: Rome, Italy.

FAO. 1991. Non-wood forest products: the way ahead. FAO Forestry Paper 97: Rome.

FAO. 1995a. Report of the expert consultation on non-wood forest products, Yogyakarta, Indonesia, 17 - 27 january 1995. Non-wood Forest Products 3. FAO: Rome.

FAO. 1995b. Non-wood forest products for rural income and sustainable forestry. Non-wood Forest Products 7. FAO: Rome.

FAO. 2005. Situation des Forêts du Monde. FAO: Rome.

FAO. 2007. Gestion durable des produits forestiers non ligneux (PFNL) dans les concessions forestières au Cameroun Note d'information. Projet MTF/CMR/025/MSC. 
FAO /COMIFAC. 2006. Gestion des ressources naturelles fournissant des produits forestiers non ligneux en Afrique Centrale. Note d'information No.4. PROJET GCP/RAF/398/GER

Fines JP, Lescuyer G, Tchatat M. 2001a. Master Management plan for the Tropenbos-Cameroon Programme research site: pre-mediation version. Tropenbos-Cameroon Document 5. Tropenbos-Cameroon Programme: Kribi Cameroun.

Fines JP, Ngibaot F, Ngono G. 2001b. A conceptual Forest Management plan for a medium size forest in Southern Camaeroon. Tropenbos-Cameroon Document 6. Tropenbos-Cameroon Programme : Kribi Cameroun.

Fokunang CN, Jiofack RB, Ngameni B, Asongalem E, Guedje NM, Kechia F, Tembe-Fokunang E, Ngoupayou J, Torimiro NJ, Gonsu KH, Sielinou V, Ndikum V, Tabi O, Ngadjui BT, Ngogang J, Asonganyi T, Abena OM, Nkongmeneck, Lohoue J, Colizzi V, Angwafor III F. 2010. Traditional Medicine: Past, Present and Future Development Prospects and Integration in the National Health System of Cameroon. Accepted for publication by African Journal of Traditional, Alternative and Complementary Medecine (AJTCAM).

Friedberg C. 1999. Les relations aux ancêtres aujourd'hui et la gestion de la forêt tropicale dans l'Indonésie de l'est. In L'Homme et la Forêt Tropicale, Bahuchet S, Bley D, Pagezy H, Vernazza-Licht N (eds). Société d'Ecologie Humaine ; 4557.

Godoy R, Lubowski R. 1992. Guidelines for the economic valuation of non-timber tropical forest products. Current Anthropology, 33: 423-433.

Guedje NM. 2002. La gestion des populations d'arbres comme outil pour une exploitation durable des Produits Forestiers Non-Ligneux: l'exemple de Garcinia lucida (Sud-Cameroun). Tropenbos-Cameroun Series 5. The
Tropenbos-Cameroon Programme, Kribi, and Université Libre de Bruxelles, Brussels.

Guedje NM, Fankap R. 2001. Utilisations traditionnelles de Garcinia lucida et Garcinia kola (Clusiaceae) au Cameroun. Syst. Geogr. Pl., 71: 747-758.

Guedje NM, Lejoly J, Nkongmeneck BA, Jonkers WBJ. 2003. Population dynamics of Garcinia lucida (Clusiaceae) in Cameroonian Atlantic forests. Forest Ecol Manag., 177: 231-241.

Guedje NM, Nkongmeneck BA, Lejoly J. 2006. Intégration des savoirs-faire locaux aux connaissances scientifiques pour une gestion participative et durable des ressources naturelles. In CommunityBased Conservation of Natural Resources in Dry and Subhumid Savannas Mayaka TB, Fotsing E (eds.). Proceedings of the Second RNSCC International Seminar, 8 February 2006, Yaounde, Cameroon; 7379.

Guedje NM, Zuidema PA, During H, Foahom B, Lejoly J. 2007. Tree bark as a nontimber forest product: The effect of bark collection on population structure and dynamics of Garcinia lucida Vesque. Forest Ecol. Manag., 240: 1-12. doi:10.1016/j.foreco.2006.09.029

Guedje CNM, Mouamfon M, Bigombé Logo P, Abéga SC, Lejoly J. 2008. Impact de la gestion socioéconomique et technique des forêts communautaires à l'échelle des économies familiales. Cas de Kompia et Kabilone (Est-Cameroun). In Gouvernance et Environnement en Afrique Centrale : le Modèle Participatif en Question, Roulet PA, Assenmaker P (eds). Musée Royal de l'Afrique Centrale: Tervuren, Belgique ; 139 157.

Harms KE, Dalling JW. 1997. Damage and herbivory tolerance through resprouting as an advantage of large seed size in tropical trees and lianas. J. Trop. Ecol., 13(4): 617-621.

Heinrich M, Barnes J, Gibbons S, Williamson EM. 2004. Fundamentals of 
Pharmacognosy and Phytotherapy. Churchill Livingstone: Spain.

Ingram V, Chupezi Tieguhong J, Nkamgnia EM, Eyebe JP, Ngawe M. 2010. Bamboo Production to Consumption System, Cameroon. Working Paper. Center for International Forestry Research (CIFOR): Bogor, Indonesia.

Jiofack T, Ayissi I, Fokunang C, Guedje N, Kemeuze V. 2009. Ethnobotany and phytomedicine of the upper Nyong valley forest in Cameroon. African Journal of Pharmacy and Pharmacology, 3(4): 144150. http://www.academicjournals.org/ ajpp

Jiofack T, Fokunang C, Guedje N, Kemeuze V, Fongnzossie E, Nkongmeneck BA, Mapongmetsem PM, Tsabang N. 2010. Ethnobotanical uses of medicinal plants of two ethnoecological regions Cameroon. International Journal of Medicine and Medical Sciences, 2(3): 6079. http://www.academicjournals.org/ ijmms

Kamtchouing P, Kahpui SM, Dzeufiet PDD, Tédong L, Asongalem EA, Dimo T. 2006. Anti-diabetic activity of methanol/methylene chloride stembark extracts of Terminalia superba and Canarium schweinfurthii on streptozotocininduced diabetic rats. Journal of Ethnopharmacology, 104: 306-309.

Kasparek M, al-Janabi S. 2008. Plantes médicinales - La diversité biologique au service de la santé. Fiche thématique. Deutsche Gesellschaft für Technische Zusammenarbeit (GTZ) GmbH.

Kuete V, Tabopda TK, Ngameni B, Nana F, Tshikalange TE, Ngadjui BT. 2010. Antimycobacterial, antibacterial and antifungal activities of Terminalia superba (Combretaceae). South African Journal of Botany, 76: 125 - 131.

Juhé-Beaulaton D, Dugast S, Tostain S, Kokou K, Alfieri C, Sanou A, Varisou S. 2005. Enjeux économiques et sociaux autour des bois sacrés et la «conservation de la biodiversité» au Bénin, Burkina
Faso et Togo. In Dynamique de la biodiversité et modalités d'accès aux milieux et aux ressources. Acte du Séminaire de l'IFB, Frejus, France; 6872.

Kokou K, Adjossou K, Klaus H. 2005. Les forêts sacrées de l'aire Ouatchi au sud-est du Togo et les contraintes actuelles des modes de gestion locale des ressources forestières », VertigO, 6(3). [En ligne], mis en ligne le 01 décembre 2005. URL : http://vertigo.revues.org/2456. Consulté le 23 juillet 2010 .

Koné B, Kalinganire A, Doumbia M. 2009. La Culture du Jujubier : un Manuel pour l'Horticulteur Sahélien. ICRAF Technical Manual no. 10. World Agroforestry Centre: Nairobi.

Laird SA. 1999. The management of forests for timber and non-wood forest products in Central Africa. In Non-Wood Forest Products of Central Africa - Current Research Issues and Prospects for Conservation and Development, Sunderland TCH, Clark LE, Vantomme P (eds). FAO : Rome; 51-60.

Leakey R. 2005. Domestication potential of Marula (Sclerocarya birrea subsp. caffra) in South Africa and Namibia: 3. Multiple trait selection. Agrofor. Syst., 64: 51-59.

Leakey RRB, Tchoundjeu Z, Schreckenberg K, Simons AJ, Shackleton S, Mander M, Wynberg R, Shackleton C, Sullivan C. 2007. Trees and markets for agroforestry tree products: targeting poverty reduction and enhanced livelihoods. In World Agroforestry into the Future, Garrity D, Okono A, Parrott MS (eds). World Agroforestry Centre: Nairobi, Kenya; 1122.

Lévêque C, Mounoudou JC. 2001. Biodiversité - Dynamique Biologique et Conservation. DUNOD: Paris, France.

Malla YB. 2000. Impact de la politique de foresterie communautaire sur les moyens d'existence et la sécurité alimentaire en milieu rural au Népal. Unasylva, 202(52): 37-45. http//www.fao.org/DOCREP/ $\mathrm{X} 7273 \mathrm{~F} / \mathrm{x} 7273 \mathrm{f0} 2 . \mathrm{htm}$ 
Mapi J. 1988. Contribution à l'étude ethnobotanique et analyses chimiques de quelques plantes utilisées en médecine traditionnelle dans la région de Nkongsamba (Moungo-Cameroun). Thèse de doctorat $3^{\text {ème }}$ cycle, Université de Yaoundé, Cameroun, p. 169.

McKey D, Linares OF, Clement CR, Hladik CM. 1996. Ressources alimentaires des forêts tropicales: une mise en perspective des tendances évolutives et de l'impact du peuplement humain. In L'Alimentation en Forêt Tropicale, Interactions Bioculturelles et Perspectives de Développement, Hladik CM, Hladik A, Pagezy H, Linares OF, Koppert GJA, Froment A (eds). UNESCO / MAB: Paris ; Vol I, 43-54.

Milol CA. 1999. Gestion des forêts communautaires au Cameroun: enjeux, stratégies de mise en place, conflits. Etude de cas. In L'Homme et la Forêt Tropicale, Bahuchet S, Bley D, Pagezy H, Vernazza-Licht N (eds). Société d'Ecologie Humaine ; 487-499.

MINEF. 1998. Recueil de Textes Officiels Relatifs à la Gestion des Forêts et de la Faune au Cameroun. MINEF : Yaoundé, Cameroun.

MINFOF. 2005. Séminaire sur les dix années de gestion forestière et faunique au Cameroun (1995 - 2005): bilan et perspectives. Yaoundé, Palais des Congres, 25 au 28 avril 2005.

MINFOF. 2006. Evaluation des progrès accomplis vers la gestion durable des forêts sur la base des PCI OAB-OIBT. MINFOF : Yaoundé, Cameroun.

Ndangalasia HJ, Bitarihob R, Delali BK, Dovie. 2007. Harvesting of non-timber forest products and implications for conservation in two montane forests of East Africa. Biological Conservation, 134: 242 - 250 .

Ndoye O, Ruiz Pérez M, Eyebe A. 1998. Les Marchés des Produits Forestiers NonLigneux dans la Zone de Forêt Humide $d u$ Cameroun. Réseau foresterie pour le Développement rural, Document du réseau 22 c. ODI : London, United Kingdom.

Ndoye O, Ruiz-Perez M, Eyebe A. 2001. NTFP markets and potential degradation of forest resources in Cameroon: the case of Garcinia lucida. ETFRN News 32: 4445.

Nepstad DC, Schwartzman S (eds). 1992. Non-timber product extraction from tropical forest: evaluation of a conservation and development strategy. Advances in Economic Botany 9.

Nepstad DC, Brown LIF, Luz L, Alechandra A, Viana V. 1992. Biotic impoverishment of Amazonian forest by tappers, loggers and cattle ranchers. In Non-Timber Products from Tropical Forests: Evaluation of a Conservation and Development Strategy, Nepstad DC, Schwartzman S (eds). Advan. Econ. Bot. 9. The New York Botanical Garden: Bronx, NY; 1-14.

Ngameni B, Watchueng J, Fekam FB, Keumedjio F, Gut J, Abegaz BM, Rosenthal PJ. 2007. Antimalaria Prenylated Chalcones from the twigs of D. Barteri var. subtriagularis, Arkivoc: 116-123.

Nguenang GM, Feteke F. 2000. Une meilleure exploitation des ressources des forêts communautaires au Cameroun: quelle option choisir? Arbres, Forêts et Comm. Rurales, 19: 36-39.

Nguinguiri JC. 1999. Les approches participatives dans la gestion des écosystèmes forestiers d'Afrique centrale: revue des initiatives existantes. CIFOR: Bogor Indonésie.

Nkongmeneck BA. 2007. Répertoire des plantes médicinales du Cameroun. Cameroon J. Ethnobot., 2: 36-46.

Nkuinkeu R. 1998. Plantes Médicinales et Exploitation Forestière. In Les Produits Non Ligneux en Afrique Centrale. Recherches Actuelles et Perspectives pour la Conservation et le Développement, Sunderland TCH, Clark LE, Vantome P (eds). FAO: Rome; 281285. 
Noumi E. 1984. Les plantes à épices, à condiments et à aromates du Cameroun. Thèse de troisième cycle, Université de Yaoundé, Yaoundé.

Oldfield S, Lusty C, MacKinven A. 1998. The World List of Threatened Trees. World Conservation Press: Cambridge.

Panayotou T, Ashton P. 1992. Not by Timber Alone: the Case for Multiple Use Management of Tropical Forests. Island Press: Covelo CA.

Pénelon A. 1996. Création d'une forêt communautaire: une expérience de décentralisation de la gestion des ressources forestières au Sud-Cameroun. Le Flamboyant, 37: 18-22.

Péres CA, Baider C, Zuidema PA, Wadt LHO, Kainer KA, Gomes-Silva DAP, Saloma o RP, Simo es LL, Franciosi ERN, Valverde FC, Gribel R, Shepard GH, Kanashiro M, Coventry P, Yu DW, Watkinson AR, Frekleton RP. 2003. Demographic threats to the sustainability of Brazil nut exploitation. Science, 302: 2112-2114.

Peters CM. 1996. The ecology and management of non-timber forest resources. World Bank Technical Paper $\mathrm{N}^{\circ}$ 322: Washington, DC, USA.

Peters CM. 1997. Exploitation Soutenue de Produits Forestiers autres que le Bois en Forêt Tropicale Humide: Manuel d'Initiation Ecologique. Programme d'Appui à la Biodiversité: Washington DC.

Peters CM, Balick MJ, Kahn F, Anderson AB. 1989. Oligarchic forests of economic plants in Amazonia: utilization and conservation of an important tropical resource. Conservation Biology, 3(4): 341-349.

Pianka ER. 1970. On $r$ - and $k$ - selection. Am. Nat., 104: 592-597.

Poulin Thériault. 1995. Schéma Directeur d'aménagement polyvalent du massif forestier de Lokoundje-Nyong. Projet "Appui Institutionnel Forestier", phase transitoire. MINEF: Yaoundé, Cameroun.
Prance GT, Balée W, Boom BM, Carneiro RL. 1987. Quantitative ethnobotany and the case for conservation in Amazonia. Conserv. Biol., 1(4): 296-310.

Rice RA, Greenberg R. 2000. Cocoa cultivation and conservation of Biological diversity. Ambio., 29(3): 167-13.

Ros-Tonen MAF. (ed). 1999. NTFP research in the Tropenbos Programme: results and perspectives. Seminar Proceedings. Tropenbos Foundation: Wageningen the Netherlands.

Ros-Tonen M, Dikjman W, van Bueren EL. 1994. Commercial and sustainable extraction of non wood forest products: why not? Draft for discussion. The Tropenbos Foundation: Wageningen, The Netherlands.

Schröder JM. 2001. NTFP use and sustainability: the example of Prunus africana in Cameroon. ETFRN News, 32: 12-14.

Secretariat of the Convention on Biological Diversity. 2009. The Convention on Biological Diversity Plant Conservation Report : A Review of Progress in Implementing the Global Strategy of Plant Conservation. CBD.

Shiembo NP. 1999. The sustainability of eru (Gnetum africanum and Gnetum buchholzianum): over-exploited nonwood forest product from the forests of Centra Africa. In Non-timber Forest Products of Central Africa - Current Research Issues And Prospects For Conservation And Development, Sunderland TCH, Clark LE, Vantomme P (eds). FAO: Rome, Italy; 61-66.

Shore KJ. 2004. L'intégration des plantes médicinales en Inde. Bulletin du CRDI, Août 2004.

Simons AJ, Leakey RRB. 2004. Tree domestication in tropical agroforestry. Agroforestry Systems, 61: 167-181.

Soehartono T, Newton AC. 2001. Conservation and sustainable use of tropical trees in the genus Aquilaria II. The impact of gahuru harvesting in Indonesia. Biol. Conserv., 97: 29-41. 
Sunderland THC, Clark LE, Vantomme P. (eds). 1999a. Non-timber Forest Products of Central Africa - Current Research Issues and Prospects for Conservation and Development. FAO: Rome Italie.

Sunderland TCH, Ngo-Mpeck ML, Tchoundjeu Z, Amougou A. 1999b. The ecology and sustainability of Pausinystalia johimbe: an over-exploited medicinal plant of the forests of Central Africa. In Non-timber Forest Products of Central Africa - Current Research Issues And Prospects For Conservation And Development, Sunderland TCH, Clark LE, Vantomme P (eds). FAO: Rome Italy; 67-77.

Tabuti JRS, Lye KA, Dhillion SS. 2003. Traditional herbal drugs of Bulamogi, Uganda: plants, use and administration. Journal of Ethnopharmacology, 88: 1944.

Tchatat M. 2001. Démarches et paramètres pour la production de bois d'œuvre dans le site du Programme Tropenbos Cameroun. In Sustainable management of African rain forest, Foahom B, Jonkers WBI, Nkwi PN, Schimidt P, Tchatat M (eds). Seminar proceedings held in Kribi, Cameroon, November 1999. Part I. Workshop. The Tropenbos Foundation: Wageningen; 105-115.

Tchatat M, Ndoye O, Nasi R. 1999. Produits forestiers autres que le bois d'oeuvre (PFAB): Place dans l'amenagement durable des forets humides d'Afrique Centrale. Serie FORAFRI, 18. CIRADForêt: France.

Tchoundjeu Z, Duguma B, Tchientcheu ML, Ngo-Mpeck ML. 1999. The domestication of indigenous agroforestry trees: ICRAF's strategy in the Humid Tropics of West and Central Africa. In Non-timber Forest Products of Central Africa - Current Research Issues And Prospects For Conservation And Development, Sunderland $\mathrm{TCH}$, Clark LE, Vantomme P (eds). FAO: Rome, Italy; 161-169.
Tchoundjeu Z, Avana ML, Leakey RRB, Simons AJ, Asaah E, Duguma B, Bell JM. 2002. Vegetative propagation of Prunus africana: Effects of rooting medium, auxin concentrations and leaf area. Agrofor. Syst., 54: 183-192.

Tchoundjeu Z, Mpeck MLN, Asaah E, Amougou A. 2004. The role of vegetative propagation in the domestication of Pausinystalia johimbe (K. Schum) a highly threatened medicinal species of West and Central Africa. Forest Ecol. Manage., 188: 175-183.

Tchoundjeu Z, Asaah EK, Anegbeh P, Degrande A, Mbile P, Facheux C, Tsobeng A, Atangana AR, Ngo-Mpeck ML, Simons AJ. 2006. Putting participatory domestication into practice in West and central Africa. Forests, Trees and Livelihoods, 16: 53-69.

TRAFFIC (North America). 1999. Medicine from US Wildlands: An Assessment of Native Plant Species Harvested in the United States for Medicinal Use and trade.

Tsague A. 1995. Etude de la filière des produits de cueillette du prélèvement à la première commercialisation. Projet API Dimako: Cameroun.

UICN. 1997. Plan Directeur d'Aménagement du Parc National de Waza. MINEF / UICN (en collaboration avec SNVCAM/WWF/CML/DGIS): $\quad$ Yaoundé, Cameroun.

UICN / Union Européenne. 1998. Les écosystèmes de forêts denses et humides d'Afrique Centrale. Actes de la deuxième conférence. UICN, Union Européenne, Royaume des Pays-Bas.

van Andel TR. 2006. Les produits forestiers autres que le bois d'œuvre - la valeur des plantes sauvages. Agrodok 39 : Wageningen.

Varquez R, Gentry AH. 1989. Use and misuse of forest-harvested fruits in the Iquitos area. Conservation Biology, 3(4): 350-361. 
Vivien J, Faure JJ. 1996. Fruitiers Sauvages d'Afrique: Espèces du Cameroun. NguilaKerou, Carnoe: France.

von Hagen B, Fight RD. 1999. Opportunities for conservation-based development of non-timber forest products in the Pacific Northwest. Abstract. General Technical Reports of the US Department of Agriculture, Forest Service, PNW-GTR473.

Warner K. 2000. Foresterie et moyens d'existence durables. Unasylva, 202(51): 3-12. http//www.fao.org/DOCREP/ X7273F/x7273f02.htm.

Whitmore TC. 1989. Canopy gaps and the two major groups of forest trees. Ecology, 70(3): 536-538.

Wickens GE. 1991. Management issues for development of non-timber forest products. Unasylva, 165(42): 3-8.
Wiersum K.F. 1996. Domestication of valuable trees species in agroforestry systems: evolutionary stages from gathering to breeding. In Domestication and commercialisation of non-timber forest products in agroforestry systems, Leakey RRB, Temu AB, Melnyk M (eds). FAO Technical Papers, Non-wood forest products 9; 147-158.

Wilkie D. 1999. CARPE and Non-Wood Forest Products. In Non-Wood Forest Products of Central Africa - Current Research Issues and Prospects for Conservation and Development, Sunderland TCH, Clark LE, Vantomme P (eds). FAO: Rome, Italy; 3-16.

Wollenberg E, Ingles A. (eds). 1998. Incomes from the Forest. Methods for the Development and Conservation of Forest Products for Local Communities. CIFOR: Bogor Indonésie. 